\title{
PEG-ON-HOLE: FUNDAMENTAL PRINCIPLES OF MOTION OF A PEG LEANING ON A HORIZONTAL HOLE EDGE
}

\author{
L.B. Chernyakhovskaya', D.A. Simakov² \\ 1 Department of Mechanics, Samara State Technical University, ul. Molodogvardeiskaya 244, 443100, Samara, \\ Russia, e-mail: tms@samgtu.ru \\ 2 Independent Researcher, Wackenroderstrasse 1, 07745 Jena, Deutschland, e-mail:dmsimak@gmail.com
}

Received: 2017.09.15

Accepted: 2017.11.01 Published: 2017.12.05

\begin{abstract}
This paper presents a solution to the problem of a peg leaning on the edge of a horizontal hole at three points. The object of the interest is the motion in the course of automated peg-in-hole assembly. The complex motion of a peg is described in two degrees of freedom. These degrees of freedom are planar motion and rotational motion. Planar motion provides parts alignment via a change of nutation angle. Rotational motion occurs around a hole axis. It is described by a precession angle. The kinematic analysis of these was performed. Based on this analysis the directions of the peg velocities at the contact points were established. The directions of forces acting on the peg at these points were also determined. The system of Dynamic Differential Equations of the generalized coordinates was derived. This system determines the equations of the peg motion and dynamic reactions. During rotational motion, the forces acting on the peg at contact points are shown to be significantly reduced. This reduction improves the conditions of parts alignment and increases the assembly quality.
\end{abstract}

Key words: cylindrical parts, three contact points, complex motion, two degrees of freedom, dynamic reactions, differential equations.

\section{INTRODUCTION}

Automation of peg-in-hole assembly remains a relevant problem of mechanical engineering due to the increasing use of robotic systems. The fundamental principles of the mechanical motion of this assembly could predict the process depending on the initial contact and relative positions of the components. The use of these fundamental principles could significantly benefit to the development of assembly devices. The development of assembly devices includes conception, design, mathematical modeling of their mechanisms and the development of process management programs.

The topic of automated peg-in-hole assembly is quite broad. The related researches could be roughly divided into two main branches: theoretical description and process control.
The works on theoretical description have included the research on the geometrical parameters of detailed motion [12], kinematic characteristics and force interactions between them. These tasks were solved for contacts at one, two or three points $[5-7,13]$. All the theoretical researches, however, consider the motion of a component depending on the change of only one parameter - a nutation angle. This is the angle between the components' axes, which represents the misalignment of the components.

Along with alignment some currently designed and utilized assembly devices use the second parameter. This is the rotational motion of a peg around its or holes' axis. In practice this rotation reduces the interaction forces between the components, and eases their alignment. The theoretical study of this case was performed using the gyroscopic approximation $[3,8,9]$. This 
approximation assumes for simplicity that the component has only one fixed point. This approximation, however, does not correctly reflect the dynamics of the motion.

The process control of the assembly automation was a point of interest in the later years. There have been the studies based on experiment $[1,2,11]$, and on mathematical modeling $[4,10]$.

This work returns back to the theoretical fundamental investigations of the problem. The original mathematical model developed in this paper broadens the understanding of geometry and of dynamics of the peg-on-hole assembly with respect to preceding papers. The new knowledge is expected to be applied process control and other practical applications easier.

One of the improvements, suggested by the mathematical model of this paper is increase of the considered dimensions. As it was mentioned, previously, only a nutation angle $\gamma=\gamma(\mathrm{t})$ describing the alignment, was taken correctly into account. This angle is one of the three Euler angles, required for the full description of mechanics and kinematics of the peg-on-hole motion.

The full set of Euler angles includes: (i) the angle of the rotation around peg's axes $\varphi=\varphi(t)$, (ii) peg rotation around the hole axis described by precession angle $\psi=\psi(\mathrm{t})$, and (iii) a planar motion of the component alignment, described by the nutation angle $\gamma=\gamma(\mathrm{t})$ between the components' axes.

The original model of this paper considers correctly two of these generalized coordinates (see Fig. 1). In addition the previously considered nutation angle $\gamma=\gamma(\mathrm{t})$, the precession angle $\psi=$ $\psi(\mathrm{t})$ is now taken into account.

Another novelty of this model is representation of the results of the analysis in the form of Dynamic Differential Equations deduced from the Lagrangian.

Dynamic Differential Equations are a powerful tool to study forces, positions and velocities during peg-on-hole alignment motion. The mechanics for a large number of alignment scenarios could be predicted using analytical and numerical solutions of the equations. This paper demonstrates the power of Dynamic Differential Equations by comparing two simple cases of motion, with one and two degrees of freedom. In this simple comparison Dynamic Differential Equations predicts the advantages of an additional precession rotation.

In the investigations of this paper, the peg and the hole are assumed to be rigid bodies of the regular geometric features with the following dimensions (see Fig. 1): $d$ is a peg diameter, $H$ is its height, $D$ is a diameter of the hole, $D-d$ is size of a guaranteed gap, $\gamma$ is an angle between the components' axis, the value of which is taken as $\gamma>\arccos \frac{d}{D}$, which corresponds to the contact between the components at three points. One of the contact points $K$ is placed between the generatrix of the peg and the hole edge in the plane, going through the axis of the components, two other contact points $B_{1}$ and $B_{2}$ are placed between the edges of both components symmetric to the plane.

The description of the positions of the elements and points requires a coordinate system. It is convenient to use the Cartesian coordinate systems for the problems of this paper. So everywhere the authors refer to a coordinate system, they always assume a Cartesian coordinate system, and the corresponding coordinates are Cartesian coordinates.

To determine the position of the peg in relation to the hole (see Fig. 1) a fixed coordinate system $O_{I} x y z$ is chosen which origin coincides with the centre $O_{l}$ of a horizontal circle of the edge of the hole. The coordinate plane $O_{l} y z$ goes through the hole axes $O_{I} z$ and the initial position of peg's

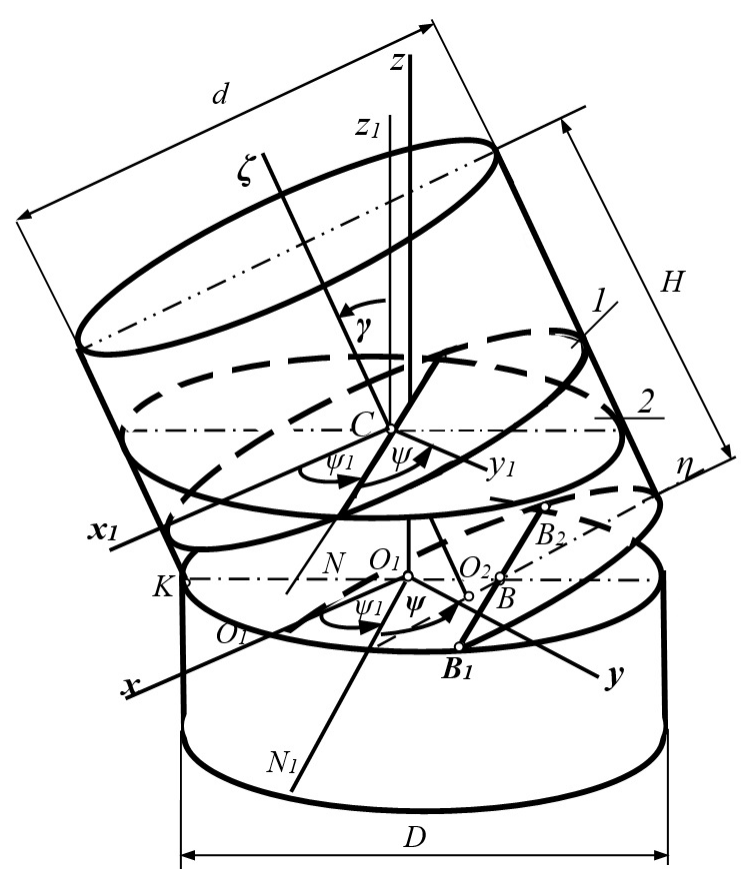

Fig. 1. Position of the peg with two degrees of freedom $\psi$ and $\gamma$. Sections 1 and 2 are the sections of the peg by the moving plane of coordinates Cx1y1 and the translation of the fixed coordinate system O1xy. The other notations are presented in the in-troduction of the paper 
axis $O_{2} \zeta$. The axes $O_{I} x$ is directed perpendicular to this plane. Angle $\gamma$ between the axes of the hole $\mathrm{O}_{I} z$ and of the peg $\mathrm{O}_{2} \zeta$, and is a nutation angle.

The moving system of coordinates $C x_{1} y_{l} z_{l}$, the axes of which are moving translationally parallel to the corresponding fixed axes, is connected with the mass centre $C$. Plane $C x_{1} y_{1}$ bisects the peg to form section 1 . Section 2 is formed by the plane going through point $C$. This plane is parallel to the base of the hole. Section 2 bisects section 1 of the peg along the line of nodes $C N$.

The angle between axis $C x_{1}$ and the line of nodes $C N$ is a precession angle $\psi_{l}$. Based on the geometric analysis it was deduced that the line of nodes is parallel to segment $B_{1} B_{2}$.

In order to simplify some required mathematical transformation, in the subsequent analysis of the rotational motion of the peg, the angle $\psi$ complementary to $\psi_{1}$ is chosen for a generalized coordinate.

Thus on further consideration of the alignment process the position of the peg leaning at the hole is characterized by coordinates $x_{C} y_{C} z_{C}$ of mass centre $C$, and two angles $\gamma$ and $\psi$.

Since the peg is not a free body, its position in the space is constrained by the bounds applied to it - three contacts points $K, B_{1}$ and $B_{2}$. Thus it has two degrees of freedom and its position is determined by two generalized coordinates: $\gamma$ and $\psi$. Therefore, to describe the motion of the peg in this case it is necessary to determine the dependence of the mass centre coordinates $x_{C} y_{C} z_{C}$, and of all its points affecting the components alignment from generalized coordinates $\gamma$ and $\psi$.

The goals of this work is divided into sections as follows:

1) kinematic analysis of the peg motion with one and two degrees of freedom,

2) determination of interaction forces at contact points and their effect on the process of alignment,

3) determination of mechanical motion rules, which defines the alignment of the parts.

\section{KINEMATIC ANALYSIS OF THE PEG MOTION}

\section{Motion in 1D: consideration of nutation angle $\gamma$}

First let us consider the change of the nutation angle $\gamma$ only. In this case the peg leaning on three points of the hole edge performs a plane-parallel motion characterized by the motion of its $A E D N$ section in its own plane (see Fig. 2) relative to $O_{l} \eta z$ coordinate system with the origin at the center the hole edge $O_{l}$. Based on the geometrical analysis it was defined that all properties of this motion are the functions of angle $\gamma$.

The distance between intersection point $O$ of the components' axes and the centers of aligning edges of the hole $O_{1}$ and of the peg $O_{2}$ (see Fig. 2) is expressed by the equations

$$
\begin{aligned}
& a_{1}=O O_{1}=\frac{d-D \cos \gamma}{2 \sin \gamma} \\
& a_{2}=O O_{2}=\frac{D-d \cos \gamma}{2 \sin \gamma} .
\end{aligned}
$$

The location of symmetrical contact points $B_{1}$ and $B_{2}$ (see Fig. 2) can be determined by the distances $S_{1}=O_{1} B$ and $S_{2}=O_{2} B$ between the aperture center $O_{1}$ of the hole or center $O_{1}$ of the pegs base on one side, and point $B$ in the middle of the section between the symmetrical contact points on the other side:

$$
\begin{gathered}
S_{1}=O_{1} B=\frac{a_{2}-a_{1} \cos \gamma}{\sin \gamma} \\
S_{2}=O_{2} B=\frac{a_{1}-a_{2} \cos \gamma}{\sin \gamma} .
\end{gathered}
$$

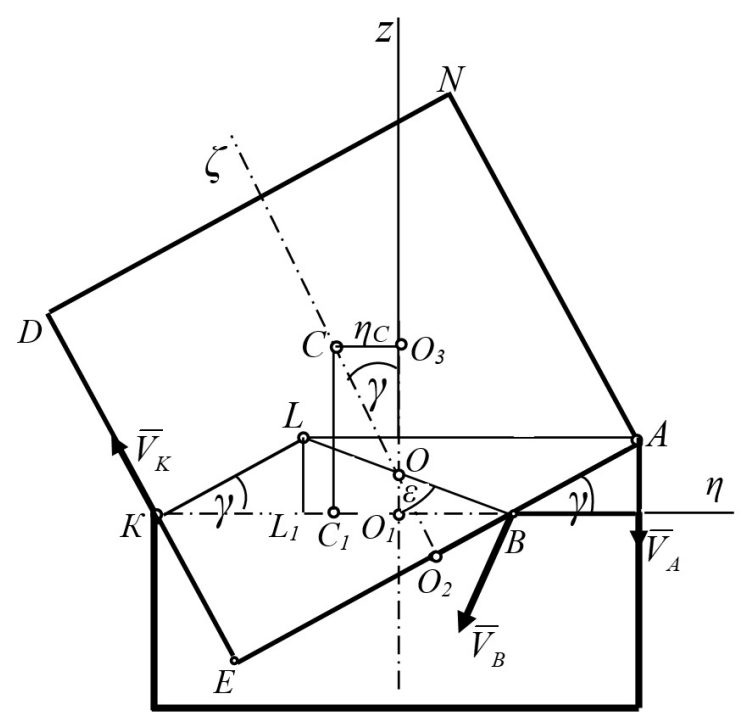

Fig.2. Velocity distribution at plane-parallel motion of the peg at points K, B, A. Contact points B1 and B2 as well as point $\mathrm{B}$ are projected to the same point in this section, and have equal velocities. The projection is denoted here as B for simplicity. See introduction and section 1 for notations 
The center of mass $C$ of the peg is convenient to consider in the $O_{l} \eta z$ coordinates system, which is located in the plane of the symmetry of the components (see Fig. 2). The coordinates of the center of mass in this system are the following

$$
\begin{gathered}
\eta_{C}=-\left(0.5 H-a_{2}\right) \sin \gamma, \\
z_{C}=\left(0.5 H-a_{2}\right) \cos \gamma+a_{1} .
\end{gathered}
$$

Point $A$ is located in the symmetry plane at the peg edge, at the diameter end overhanging maximally above the hole (see Fig. 2). Its coordinate in the same coordinate system are

$$
\eta_{A}=S_{1}+\left(\frac{d}{2}-S_{2}\right) \cos \gamma
$$

where: $S_{2}$ defined in (2) is the distance between the peg edge centre and the intersection of its most overhanging diameter with the hole edge.

The first expression in (4) could be simplified with the following result: $\eta \mathrm{A}=0.5 \mathrm{D}$. The obtained value means that point $A$ moves along the straight line coinciding with the generatrix of the hole internal surface. Therefore, its velocity for any value of angle $\gamma$ is directed vertically downwards.

In addition, the velocity direction of another peg point is known. It is that of contact point $K$ located in the components plane of symmetry. The velocity is directed along its generatrix (see Fig. 2). Knowledge of the velocities at these two points allows determination of the location of their instantaneous center $L$. It is an intersection point of the perpendiculars of the $\bar{V}_{A}$ and $\bar{V}_{K}$ velocities. The coordinates of $L$ are equal to

$$
\eta_{L}=-S_{1}, \quad z_{L}=2 a_{1} \text {. }
$$

Thus the direction of the velocity of point $B$ during plane-parallel motion of the peg can be determined. According to the definition of an instantaneous velocity centre, the velocity direction is perpendicular to $B L$ section (see Fig. 2). Due to geometry this section goes through the intersection point $\mathrm{O}_{3}$ of the axes of the peg and the hole.

The instantaneous velocity center $L$ is related to the nutational motion with angle $\gamma$. Thus, the velocities of $B$ and $K$ points could be found correspondingly as

$$
V_{B}^{\gamma}=B L \dot{\gamma}, V_{K}^{\gamma}=K L \dot{\gamma},
$$

where: $B L=2 \sqrt{a_{1}^{2}+S_{1}^{2}}, K L=\frac{2 a_{1}}{\sin \gamma}$ are the distances of these points from the instantaneous velocity center (see Fig. 2), and $\dot{\gamma}$ is the angular velocity of the hole rolling nutational motion.

During planar motion points $B_{1}$ and $B_{2}$ are located on one straight line going through $B$ point perpendicular to the components' symmetry plane, Thus, their velocities are parallel to the velocity of $B$ point and the values of all three velocities are equal, i.e. the velocities are equal $\bar{V}_{B 1}^{\gamma}=\bar{V}_{B 2}^{\gamma}=\bar{V}_{B}^{\gamma}$.

\section{Motion in 2D: consideration of nutation angle $\gamma$ and of precession angle $\psi$}

Now the peg motion determined by two generalized coordinates of $\gamma$ and $\psi$ is considered. Given this motion the coordinates of center of mass take the following values (see Fig. 3, where $O C$ is the peg axis)

$$
\begin{aligned}
& x_{C}=\eta_{C} \cos \psi=-\left(0.5 H-a_{2}\right) \sin \gamma \sin \psi ; \\
& y_{C}=\eta_{C} \sin \psi=-\left(0 . H-a_{2}\right) \sin \gamma \cos \psi ; \\
& z_{C}=\left(0.5 H-a_{2}\right) \cos \gamma+a_{1} .
\end{aligned}
$$

The coordinates of $K, B_{l}$ and $B_{2}$ contact points, in fixed coordinates (see Fig. 4), are equal to

$$
\begin{aligned}
& x_{K}=-0.5 D \sin \psi ; \\
& y_{K}=-0.5 D \cos \psi ; \\
& z_{K}=0 .
\end{aligned}
$$

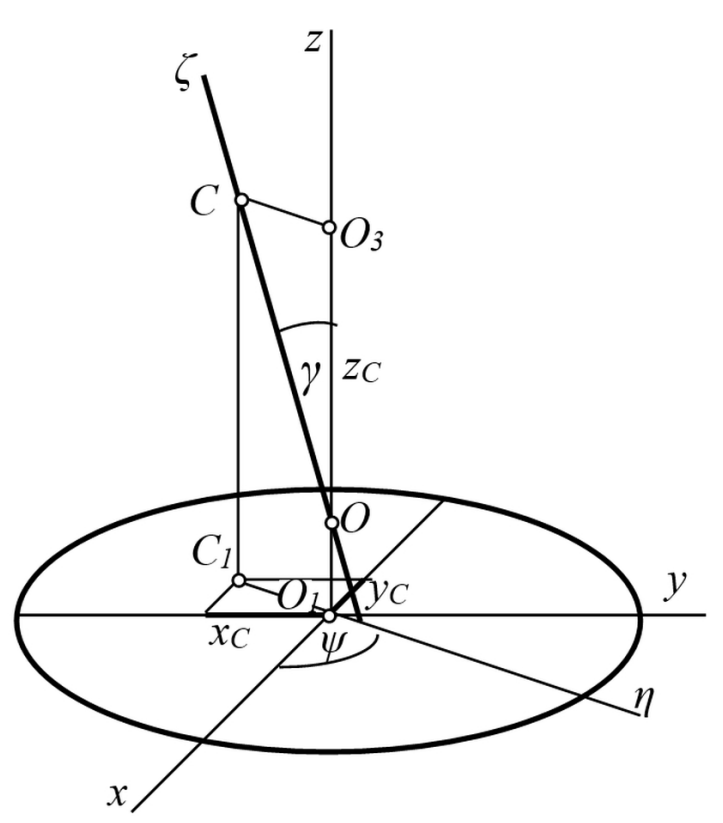

Fig. 3. Coordinates of mass center in fixed system of coordinates shown at the peg axis and the hole aperture 


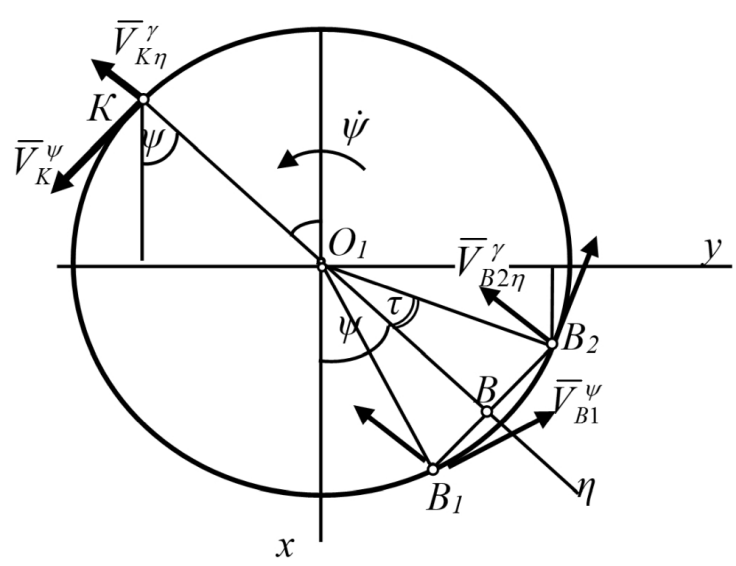

Fig. 4. Coordinates and velocities of the peg at contact points, viewed from above the peg. See the notations at section 1

$$
\begin{aligned}
& x_{B 1}=0.5 D \cos (\psi-\tau) ; \\
& y_{B 1}=0.5 D \sin (\psi-\tau) ; \\
& z_{B 1}=0 . \\
& x_{B 2}=0.5 D \sin (\psi-\tau) ; \\
& y_{B 2}=0.5 \cos (\psi-\tau) ; \\
& z_{B 2}=0 .
\end{aligned}
$$

The velocities of the rotational movement of all contact points are located in the horizontal plane, are directed tangentially to the edge of the hole aperture (see Fig. 4), and are equal to value of $V_{K}^{\psi}=V_{B 1}^{\psi}=V_{B 2}^{\psi}=0.5 D \dot{\psi}$, where $\dot{\psi}$ is the precession angular velocity, which is the angular velocity of the peg rotation around the hole axis.

The projections of absolute velocities at the contact points $\bar{V}_{K}, \quad \bar{V}_{B 1}, \quad \bar{V}_{B 2}$ to the $O_{l} \eta$ axis are equal (see Fig.2 and Fig. 4) to the sum of projections of the velocities components

$$
\begin{aligned}
& V_{K 1}^{\eta}=-K L \dot{\gamma} \sin \gamma ; \\
& V_{B 1}^{\eta}=-B L \dot{\gamma} \cos \varepsilon+0.5 D \dot{\psi} \cos \tau ; \\
& V_{B 2}^{\eta}=-B L \dot{\gamma} \cos \varepsilon-0.5 D \dot{\psi} \sin \tau ;
\end{aligned}
$$

The projections of the absolute velocities of $\bar{V}_{K}, \bar{V}_{B 1}, \bar{V}_{B 2}$ to the fixed coordinate axes are equal to

$$
\begin{aligned}
& V_{K 1}^{x}=-K L \dot{\gamma} \sin \gamma \sin \psi+0.5 D \dot{\psi} \cos \psi ; \\
& V_{K 1}^{y}=-K L \dot{\gamma} \sin \gamma \cos \psi-0.5 D \dot{\psi} \sin \psi ; \\
& V_{K 1}^{z}=K L \dot{\gamma} \cos \gamma . \\
& V_{B 1}^{x}=-B L \dot{\gamma} \cos \varepsilon \cos \psi-0.5 D \dot{\psi} \sin (\psi-\tau) ; \\
& V_{B 1}^{y}=-B L \dot{\gamma} \cos \varepsilon \sin \psi+0, D \dot{\psi}(\cos \psi-\tau) ; \\
& V_{B 1}^{z}=-B L \dot{\gamma} \sin \varepsilon .
\end{aligned}
$$

$$
\begin{aligned}
& V_{B 2}^{x}=-B L \dot{\gamma} \cos \varepsilon \cos \psi-0.5 D \dot{\psi} \cos (\psi-\tau) ; \\
& V_{B 2}^{y}=-B L \dot{\gamma} \cos \varepsilon \sin \psi+0.5 D \dot{\psi} \sin (\psi-\tau) ; \\
& V_{B 2}^{z}=-B L \dot{\gamma} \cdot \sin \varepsilon .
\end{aligned}
$$

In these expressions (see Fig. 4): $\sin \tau=\frac{b}{0.5 D}, \cos \tau=\frac{S_{1}}{0.5 D}, b=B B_{1}=\sqrt{0.25 D^{2}-S_{1}^{2}}$.

The obtained values allow determination of the direction of friction forces at contact points with the help of direction cosines, the values of which are opposite in sign to the direction cosines of absolute velocities at corresponding points. Thus, direction cosines of the angles between by friction forces at points $K, B_{I}$ and $B_{2}$ and $O_{I} \eta$ axis are equal to

$$
\begin{aligned}
& \cos v_{K}=-\frac{V_{K \eta}}{V_{K}} ; \\
& \cos v_{B 1}=-\frac{V_{B 1 \eta}}{V_{B 1}} ; \\
& \cos v_{B 2}=-\frac{V_{B 2 \eta}}{V_{B 2}} .
\end{aligned}
$$

Direction cosines of friction forces at contact points with fixed coordinates may be determined in a similar way.

\section{ANALYSIS OF FORCES ACTING AT THE PEG AT CONTACT POINTS}

During the motion, the peg is affected at each contact point (see Fig. 6) by four kind of forces: (i) the assembling exertion, (ii) gravity, (iii) normal reaction, and (iv) the friction force. The friction force hinders the alignment course.

The directions of normal reactions at contact points depend on the type of interaction. In the course of alignment (see Fig. 5) the peg leans on the edge of the hole at point $\mathrm{K}$ with its generatrix. The normal reaction $\bar{N}_{K 1}$ at this point is directed perpendicular to the generatrix. This reaction is situated inside the plain of the components' symmetry. The projections of $\bar{N}_{K 1}$ reaction to coordinates $O \eta$ and $O z$ (Fig.5) are equal to

$$
\begin{aligned}
& N_{K}^{\eta}=N_{K} \cos \gamma ; \\
& N_{K}^{z}=N_{K} \cos \gamma ;
\end{aligned}
$$

The normal reaction at each of the symmetric contact points $B_{1}$ and $B_{2}$ is located in two 
planes simultaneously: (i) a plane normal to the peg edge and going through the axis of peg, and (ii) a plane normal to the hole edge and going through the axis of hole (see Fig. 5). The line of their intersection connects a contact point to point $O$ of the intersection of the components' axes. Thus, the normal reactions acting on the peg are directed along the straight lines going through $B_{1} O$ and $B_{2} O$. They are connecting the contact points $B_{1}$ and $B_{2}$ with point of intersection $O$ of the peg and the hole axes.

The projections of the normal reactions at the symmetric contact points (Fig. 5 and Fig. 6) to the fixed coordinates are equal to

$$
\begin{gathered}
N_{K 1}^{x}=N_{K 1} \cos \gamma \cos \psi ; \\
N_{K 1}^{y}=N_{K 1} \cos \gamma \sin \psi ; \\
N_{K 1}^{z}=N_{K 1} \sin \gamma ; \\
N_{B 1}^{x}=-N_{B 1} \cos v \cos (\psi-\tau) ; \\
N_{B 1}^{y}=-N_{B 1} \cos v \sin (\psi-\tau) ; \\
N_{B 1}^{z}=N_{B 1} \sin v ; \\
N_{B 2}^{x}=-N_{B 2} \cos v \sin (\psi-\tau), \\
N_{B 2}^{y}=-N_{B 2} \cos v \cos (\psi-\tau) ; \\
N_{B 2}^{z}=N_{B 2} \sin v ;
\end{gathered}
$$

The angle $v$ in these expressions is the angle between sides $O_{1} B_{1}$ and $O B_{1}$ in triangle $O_{1} B_{1} O_{3}$ (see Fig. 5).

The value of friction force $F$ acting on the peg at a contact point is defined by the laws of Amontons and Coulomb, and its modulus is equal to $f N$, where $f$ is a friction coefficient and $N$ is the value of the normal reaction at the corresponding point.

The directions of the friction forces are opposite to their absolute velocities at the corresponding points. The projections of these forces on the coordinate axes are determined using direction cosines. The values of these direction cosines have the opposite sign to the direction cosines of the absolute velocities. Therefore, the direction cosines of the force frictions at the points $K, B_{1}$ and $B_{2}$ are equal to

$$
\begin{aligned}
& \cos \alpha_{K}=-\frac{V_{K x}}{\left|\bar{V}_{K}\right|} ; \\
& \cos \beta_{K}=-\frac{V_{K y}}{\left|\bar{V}_{K}\right|} ; \\
& \cos \lambda_{K}=-\frac{V_{K z}}{\left|\bar{V}_{K}\right|} ;
\end{aligned}
$$

$$
\begin{aligned}
& \cos \alpha_{B 1}=-\frac{V_{B 1 x}}{\left|\bar{V}_{B 1}\right|} \\
& \cos \beta_{B 1}=-\frac{V_{B 1 y}}{\left|\bar{V}_{B 1}\right|} ; \\
& \cos \lambda_{B 1}=-\frac{V_{B 1 z}}{\left|\bar{V}_{B 1}\right|} ; \\
& \cos \alpha_{B 2}=-\frac{V_{B 2 x}}{\left|\bar{V}_{B 2}\right|} \\
& \cos \beta_{B 2}=-\frac{V_{B 2 y}}{\left|\bar{V}_{B 2}\right|} ; \\
& \cos \lambda_{B 2}=-\frac{V_{B 2 z}}{\left|\bar{V}_{B 2}\right|},
\end{aligned}
$$

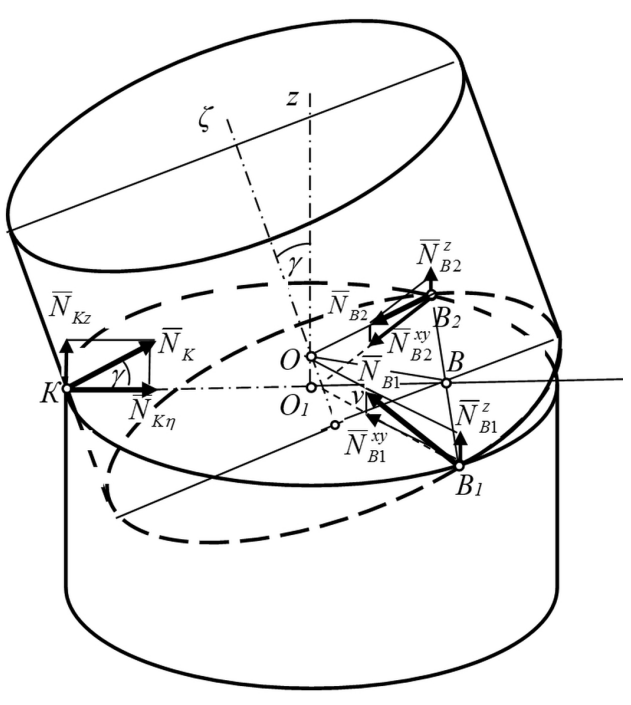

Fig. 5. Directions of normal reactions at con-tact points. See notations at section 2

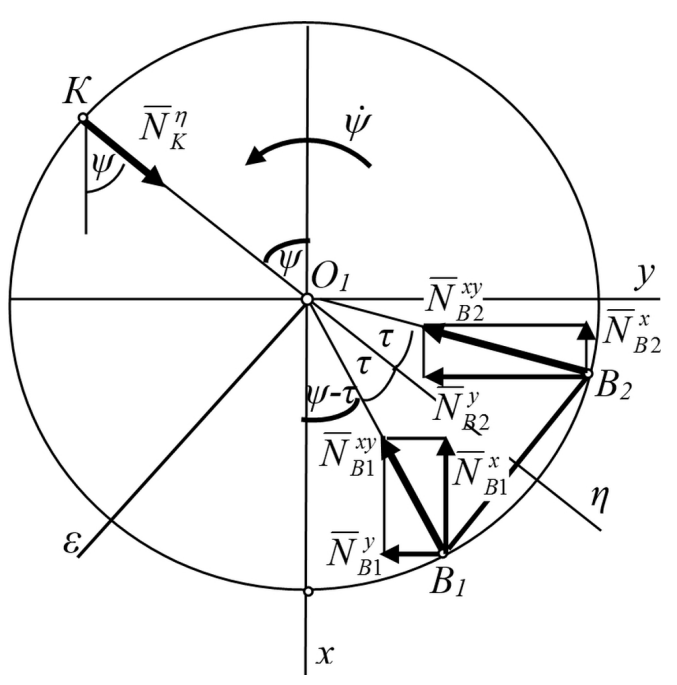

Fig. 6. Projections of normal reactions to $\mathrm{O}_{1} \mathrm{xy}$ plane, view from above at the peg. For notations see section 2 
where the values of the velocity projections to the coordinate axes are defined by the values from (11-13).

The direction cosines of the angles made by the friction forces with axis $O_{I} \eta$ at points $K, B_{l}$ and $B_{2}$ are equal to

$$
\begin{aligned}
& \cos v_{K}=-\frac{V_{K \eta}}{V_{K}} ; \cos v_{B 1}=-\frac{V_{B 1 \eta}}{V_{B 1}} ; \\
& \cos v_{B 2}-\frac{V_{B 2 \eta}}{V_{B 2}} ;
\end{aligned}
$$

where the projections of the velocities on axis $O_{I} \eta$ are defined by the values` (10).

\section{DYNAMIC DIFFERENTIAL EQUATIONS OF THE PEG MOTION}

To determine the position of the peg with two orders of freedom, Lagrangian differential equations are defined.

$$
\frac{d}{d t} \frac{\partial T}{\partial \dot{\gamma}}-\frac{\partial T}{\partial \gamma}=Q_{\gamma} ; \quad \frac{d}{d t} \frac{\partial T}{\partial \dot{\psi}}-\frac{\partial T}{\partial \psi}=Q_{\psi} .
$$

In these equations the kinetic energy $T$ of the peg's complex motion, is equal to the sum of kinetic energies of a planar motion, the main axis of which coincides with the symmetry axis of the components, and of a rotational movement around the hole axis $O_{l} z$. This energy is defined by the expression

$$
T=\frac{m V_{C \gamma}^{2}}{2}+\frac{I_{C \varepsilon} \dot{\gamma}^{2}}{2}+\frac{I_{O_{1}} \dot{\psi}^{2}}{2},
$$

where: $m$ is the peg mass;

$\mathrm{V}_{\mathrm{Cy}}$ - is the velocity of its mass centre at planar motion, which is defined through

$$
V_{C \gamma}^{2}=\left[\left(\frac{d \eta_{c}}{d \gamma}\right)^{2}+\left(\frac{d z_{C}}{d \gamma}\right)^{2}\right] \dot{\gamma}^{2} ;
$$

$I_{C \varepsilon}=\frac{m}{12}\left(3 R^{2}+H^{2}\right)$ is a peg inertia moment in relation to its central axis $C \varepsilon$ going through center of mass perpendicular to the symmetry plane;

$I_{O_{1} z}$ is a peg inertia moment in relation to the hole axis $O_{I} z$, and $\dot{\psi}$ is the angular velocity of the rotational movement.

During the alignment process, the inertia moment $I_{O_{1} z}$ of the peg in relation to the hole axis $O_{I} z$ depends on angle $\gamma$ and is defined by the expression

$$
\begin{gathered}
I_{O_{1} z}=I_{C \varepsilon} \sin ^{2} \gamma+I_{C \zeta} \cos ^{2} \gamma+m \eta_{C}^{2}(23) \\
\text { where } I_{C \zeta}=\frac{m R^{2}}{2} \text {, and } I_{C \varepsilon}=\frac{m}{12}\left(3 R^{2}+H^{2}\right)
\end{gathered}
$$
are the main central moments of the pegs inertia.

After the substitution of the expression of kinetic energy into Lagrange equation and the consequent transformations, the left parts of the Lagrange equations are reduced to the form

$$
\begin{aligned}
& \frac{d}{d t} \frac{\partial T}{\partial \dot{\gamma}}-\frac{\partial T}{\partial \gamma}=m\left[\left(\frac{d \eta_{C}}{d \gamma}\right)^{2}+\left(\frac{d z_{C}}{d \gamma}\right)^{2}\right] \ddot{\gamma}+ \\
& +I_{C \varepsilon} \ddot{\gamma}+m\left(\frac{d \eta_{C}}{d \gamma} \frac{d^{2} \eta_{C}}{d \gamma^{2}}+\frac{d z_{C}}{d \gamma} \frac{d^{2} z_{C}}{d \gamma^{2}}\right) \dot{\gamma}^{2}- \\
& -\left[m\left(\frac{d \eta_{C}}{d \gamma} \frac{d^{2} \eta_{C}}{d \gamma^{2}}+0,5 \frac{d I_{C z}}{d \gamma}\right] \dot{\psi}^{2} ;\right. \\
& \quad \frac{d}{d t} \frac{d T}{d \dot{\psi}}-\frac{d T}{d \psi}=\left(m \eta_{C}^{2}+I_{C z}\right) \ddot{\psi}+ \\
& +\left(2 m \eta_{C} \frac{d \eta_{C}}{d \gamma}+\frac{d I_{C z}}{d \gamma}\right) \dot{\gamma} \dot{\psi} .
\end{aligned}
$$

In the first Lagrange equation, the generalized force $Q_{\gamma}$ equals the sum of moments of all the forces in relation to instantaneous rotational axis $L l$ (see Fig. 8) going through point $L$ perpendicular to the plane of the components' symmetry. The sum of moments is composed of moment $M$ created by the assembling forces, the moment of gravity $M_{m g}$ and the sums of the friction forces at the contact points. The moments of the normal reactions in relation to point $L$ equal zero, because their lines of action cross the instantaneous axis of rotation.

The moment of the gravity force in relation to the instantaneous axis (see Fig. 8) equals $M_{m g}=m_{L}(m \bar{g})=-m g\left(S_{1}-\eta_{C}\right)$.

To determine the moments of the friction forces in relation to the instantaneous axis $L l$ the new coordinate axes are introduced: (i) axis $L \eta_{1}$ parallel to axes $O_{l} \eta$, and (ii) axis $L z_{l}$ parallel to axes $O_{I} z$ (see Fig. 7). The moment of the friction force at each contact point $K, B_{1}, B_{2}$ is determined by a formula $m_{L l}(\bar{F})=\eta_{1} F_{z 1}-z_{1} F_{\eta 1}$, where $F_{z l}$ and $F_{\eta I}$ are the projection of friction forces on coordinate axes $L z_{l}$ and $L \eta_{1}$ (see $\bar{F}_{K}$ and $\bar{F}_{B}$ at Fig. 8), while $\eta_{1}$ and $\mathrm{z}_{1}$ are the coordinates of the points of contact $K$ and $B$ in this coordinate system. Here $B$ is the general notation for the two contact points $B_{1}$ and $B_{2}$. 
Projection of the friction forces $F_{z I}=F_{z}$, and $F_{\eta 1}=F_{\eta}$ are found with use of direction cosines, the values of which are presented by the expressions $(18,19,20)$ and $(21)$.

Coordinates of the points of contact $K, B_{1}$ and $B_{2}$ in the coordinate system related to the instantaneous velocity centre are shown in Fig. 7 and equal

$$
\begin{aligned}
& \eta_{1 K}=-2 a_{1} \operatorname{ctg} \gamma ; \\
& z_{1 K}=-2 a_{1} ; \\
& \eta_{1 B 1}=\eta_{1 B 2}=2 S_{1} ; \\
& z_{1 B 1}=z_{1 B 2}=-2 a_{1} ;
\end{aligned}
$$

After the substitution of all the values and the subsequent transformations, the first Lagrange equation is reduced to the form

$$
\begin{aligned}
& m\left[\left(\frac{d \eta_{C}}{d \gamma}\right)^{2}+\left(\frac{d z_{C}}{d \gamma}\right)\right] \ddot{\gamma}+I_{\zeta} \ddot{\gamma}+ \\
& +m\left[\left(\frac{d \eta_{C}}{d \gamma} \frac{d^{2} \eta_{C}}{d \gamma^{2}}+\frac{d z_{C}}{d \gamma} \frac{d^{2} z_{C}}{d \gamma^{2}}\right) \dot{\gamma}^{2}-\right. \\
& \left.-m\left(\frac{d \eta_{C}}{d \gamma} \frac{d^{2} \eta_{C}}{d \gamma^{2}}\right)+0,5 \frac{d I_{z}}{d \gamma}\right] \dot{\psi}^{2}= \\
& =M_{\gamma}+m g\left(\eta_{C}-S_{1}\right)- \\
& -f N_{K}\left(\cos \gamma \cos \lambda_{K}-\cos v_{K}\right) \\
& +f N_{B 1} 2\left(S_{1} \cos \lambda_{B 1}+a_{1} \cos v_{B 1}\right) \\
& +f N_{B 2} 2\left(S_{1} \cos \lambda_{B 2}-a_{1} \cos v_{B 2}\right) ;
\end{aligned}
$$

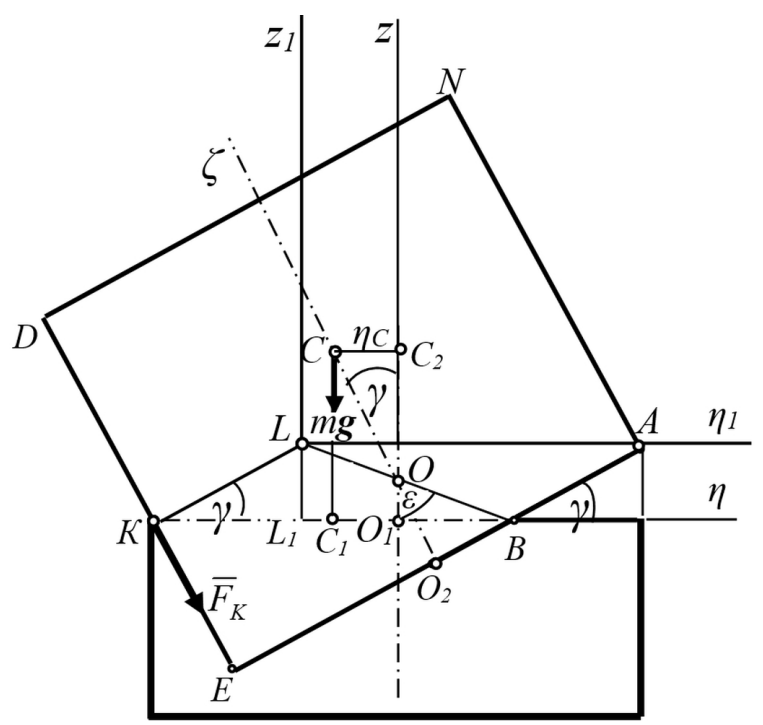

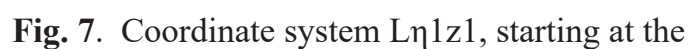
instantaneous velocity centre L. See further notation in section 2
In the second Lagrange equation the generalized force $Q_{\psi}$ equals the sum of the moments of the assembling forces $M_{\psi}$ and the moments of friction forces in relation to the hole axis $O_{I}$. Moments of the normal reactions and of gravity force in relation to the same axis equal zero.

Moments of friction forces at each contact point $K, B_{1}, B_{2}$ in relation to axis $O_{I} z$ were defined by the formula $m_{z}(\bar{F})=x F_{y}-y F_{x}$, where $x$ and $y$ are the coordinates of the points of contact in the fixed coordinate system $O_{I} x y z$, the values of which are determined with the equations (7-9). The projections of the friction forces $F_{x}$ and $F_{y}$ onto the fixed coordinate axes are determined with the help of the direct cosines, the expressions of which are determined with expressions (18-20).

The second Lagrange equation after the substitution of all the values and the consequent transformations is reduced to the following form:

$$
\begin{aligned}
& \left(m \eta_{C}^{2}+I_{z}\right) \ddot{\psi}+\frac{d I_{z}}{d \gamma} \dot{\gamma} \dot{\psi}= \\
& M^{\psi}-f N_{K} 0.5 D\left(\cos \psi \cos \beta_{K}-\sin \psi \cos \alpha_{K}\right)+ \\
& +f N_{B 1} 0.5 D\left[\cos (\psi-\tau) \cos \beta_{B 1}-\sin (\psi-\tau) \cos \alpha_{B 1}\right]+ \\
& +f N_{B 2} 0.5 D\left[\sin (\psi-\tau) \cos \beta_{B 2}-\cos (\psi-\tau) \cos \alpha_{B 2}\right] .
\end{aligned}
$$

Except generalized coordinates $\gamma$ and $\psi$ equations (26) and (27) contain three normal reactions $\bar{N}_{K}, \bar{N}_{\hat{A} 1}$ and $\bar{N}_{\hat{A} 2}$ with unknown value applied at the points at which the two components touch. To determine these reactions, it is necessary to set up the differential equations for the motion of the peg's center of mass:

$$
\begin{aligned}
& m \frac{d^{2} x_{C}}{d t^{2}}=\sum F_{k x}, \quad m \frac{d^{2} y_{C}}{d t^{2}}=\sum F_{k y}, \\
& m \frac{d^{2} z_{C}}{d t^{2}}=\sum F_{k z} .
\end{aligned}
$$

In these equations the coordinates of the peg's center of mass are determined by equations (6). The right sides of equations (28) represent the projections on the fixed coordinates of all forces applied to the peg: (i) assembly effort, the geometrical sum of which is equal to $\bar{F}^{\text {as }}$, (ii) gravity force $m \bar{g}$, normal reactions $\bar{N}_{K}, \bar{N}_{\hat{A} 1}$ and $\bar{N}_{\hat{A} 2}$, and the friction forces related to the normal reactions by Coulomb's law. The projections of normal reactions are determined by equations (15-17), and the projections of friction forces are expressed through the direction cosines.

The equations of center of mass movement after substituting all the values and transformations are reduced to the form 


$$
\begin{aligned}
m\left[\frac{d \eta_{C}}{d \gamma} \cos \psi \ddot{\gamma}-\eta_{C} \sin \psi \ddot{\psi}+\frac{d^{2} \eta_{C}}{d \gamma^{2}} \cos \psi \dot{\gamma}^{2}-\right. \\
\left.-\eta_{C} \cos \psi \dot{\psi}^{2}-2 \frac{d \eta_{C}}{d \gamma} \sin \psi \dot{\gamma} \dot{\psi}\right]=F_{x}^{a s}+ \\
+N_{K}\left(\cos \gamma \cos \psi+f \cos \alpha_{K}\right)- \\
-N_{B 1}\left[\cos v \cos (\psi-\tau)-f \cos \alpha_{B 1}\right]- \\
-N_{B 2}\left[\cos v \sin (\psi-\tau)-f \cos \alpha_{B 2}\right] ; \\
m\left[\frac{d \eta_{C}}{d \gamma} \sin \psi \ddot{\gamma}+\eta_{C} \cos \psi \ddot{\psi}+\frac{d^{2} \eta_{C}}{d \gamma^{2}} \sin \psi \dot{\gamma}^{2}\right. \\
\left.-\eta_{C} \sin \psi \dot{\psi}^{2}+2 \frac{d \eta_{C}}{d \gamma} \cos \psi \dot{\gamma} \dot{\psi}\right]=F_{y}^{a s}+ \\
+N_{K}\left(\cos \gamma \sin \psi+f \cos \beta_{K}\right)- \\
-N_{B 1}\left[\cos v \sin (\psi-\tau)-f \cos \beta_{B 1}\right]- \\
-N_{B 2}\left[\cos v \cos (\psi-\tau)-f \cos \beta_{B 2}\right] ; \\
\\
\quad m\left(\frac{d^{2} z_{C}}{d \gamma^{2}} \dot{\gamma}^{2}+\frac{d z_{C}}{d \gamma} \ddot{\gamma}\right)= \\
\quad-m g+N_{K}\left(\sin \gamma+f \cos \lambda_{K}\right)+ \\
+N_{B 1}\left(\sin v+f \cos \lambda_{B 1}\right)+ \\
+N_{B 2}\left(\sin v-f \cos \lambda_{B 2}\right) .
\end{aligned}
$$

The obtained differential equations (12), (27), (29), (30), (31) are the so-called Dynamical Differential Equations. They represent a mathematical model of the alignment process of cylindrical parts. This model allows to determine the laws of mechanical motion and interaction forces for peg movement with two degrees of freedom.

The Dynamical Differential Equations permit to describe thoroughly the peg-on-hole alignment motion. The mechanics for large number of the alignment scenarios can be completely solved using their analytical and numerical solutions. This paper demonstrates the power of the Dynamical Differential Equations by comparing two simple cases of rotational movement and deducing important results about the system dynamics from them. The simple cases are: (i) twodimensional motion with two degrees of freedom, where both degrees of freedom evolve linearly $\gamma=\gamma(t)=C_{\gamma} t$ and $\psi=\psi(t)=C_{\psi} t$ and (ii) motion with one degree of freedom evolving also linearly: $\gamma=\gamma(t)=C_{\gamma} t$. This simple comparison could bring to important results, showing the potential for analysis of more complicated tasks.

In the case (ii) the dynamic reactions are determined for the system of forces located in the components' symmetry plane, affecting the peg and resulting to the force $\bar{F}^{c \sigma}$ and the pair of forces with the momentum $M_{\gamma}$ In this case the peg performs a planar movement, its position is determined by one generalized coordinate $\gamma$, and the position of its center of mass is determined by two coordinates $-\eta_{C}$ and $z_{C}$ in the coordinate system $O_{l} \eta z$ (see Fig. 8).

For the determination of the peg position in the second case, it is necessary to use three equations: The Lagrange equation for determining the generalized $\gamma$ coordinate, and two differential equations for center of mass movement to determine the dynamic reactions. These equations are obtained from the differential equations by exclusion of the parameters related to generalized coordinate $\psi$, i.e. by substituting $\psi=0$ and $\dot{\psi}=0$.

During transformations of the equations it should be noted that in planar movement all the active forces are aligned along the plane. Normal reactions (see Fig. 9) at symmetrical points $B_{l}$ and $B_{2}$ have equal absolute values and are reduced to the resultant $\bar{N}_{B}$, which is located in the symmetry plane of the components and is applied to the middle of $B_{1} B_{2}$ section (at point $B$ ), and is directed along the straight line going through point $O$ of the intersection of the axes of the components. It can be shown geometrically that points $B, O$ and $L$ are in one straight line. Thus, the line of the direction of the resultant also points through the

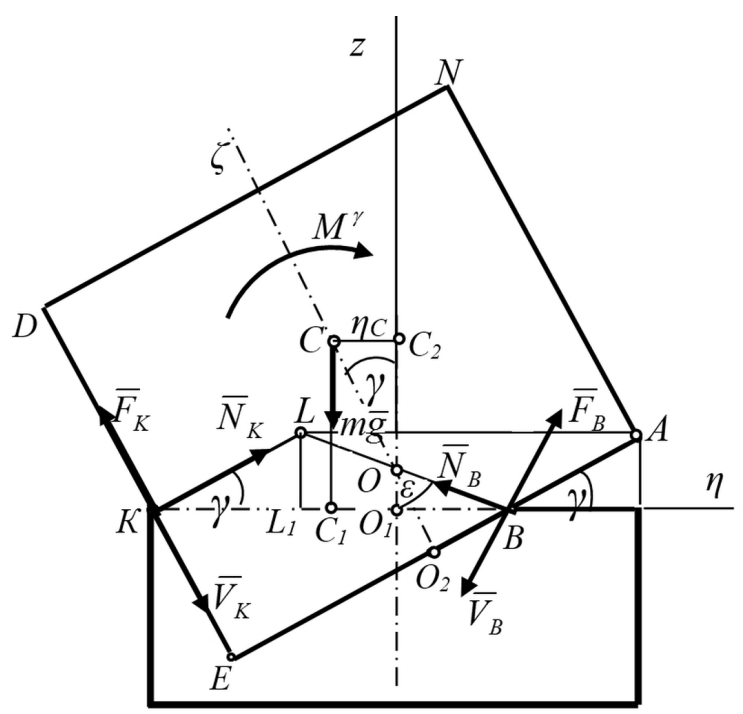

Fig. 8. Directions of normal reactions $\mathrm{N}$ and fric-tion forces $\mathrm{F}$ together with velocities $\mathrm{V}$ of pla-nar movement at contact points $\mathrm{K}$ and $\mathrm{B}$. Addi-tionally, the center of mass of the peg $\mathrm{C}$ and the instantaneous rotation center $\mathrm{L}$ with their pro-jections to axes $\mathrm{O} 1 \mathrm{z}$ and $\mathrm{O} 1 \eta$ as well as assem-bling force moment $\mathrm{M} \gamma$.

Other notations are in the text of the paper 
instantaneous center $L$ of the velocities. The value of the resultant (see Fig. 8) is equal to:

$$
N_{B}=2 N_{B 1} \sin \alpha,
$$

where: $N_{B 1}=N_{B 2}$ are the reactions at symmetrical contact points $B_{1}$ and $B_{2}$, and $\alpha$ is an angle between segments $O B_{1}$ and $B_{1} B_{2}$.

Friction forces at symmetrical contact points are directed opposite to the velocities at these points, and are equal in value and reduced to the resultant applied at point $B$, equal to: $\mathrm{F}_{\mathrm{B}}=2 \mathrm{fN}_{\mathrm{B} 1}$

After all transformations the Dynamical Differential Equations of the peg movement are considered under the effect of only one moment $M_{\gamma}$ and have the following form:

$$
\begin{gathered}
m\left[\left(\frac{d \eta_{C}}{d \gamma}\right)^{2}+\left(\frac{d z_{C}}{d \gamma}\right)^{2}\right] \ddot{\gamma}+I_{\zeta} \ddot{\gamma}+ \\
+m\left(\frac{d \eta_{C}}{d \gamma} \frac{d^{2} \eta_{C}}{d \gamma^{2}}+\frac{d z_{C}}{d \gamma} \frac{d^{2} z_{C}}{d \gamma^{2}}\right) \dot{\gamma}^{2}= \\
=M_{\gamma}+m g\left(y_{L}-y_{C}\right)- \\
-2 f N_{B 1} B L-f N_{K} K L . \\
m\left(\frac{d \eta_{C}}{d \gamma} \ddot{\gamma}+\frac{d^{2} \eta_{C}}{d \gamma^{2}} \dot{\gamma}^{2}\right)=F_{\eta}^{c \sigma}- \\
-2 N_{B 1}(\sin \alpha \sin \varepsilon-f \cos \varepsilon)+ \\
+N_{K}(\cos \gamma+f \sin \gamma) \\
m\left(\frac{d z_{C}}{d \gamma} \ddot{\gamma}+\frac{d^{2} z_{C}}{d \gamma^{2}} \dot{\gamma}^{2}\right)=F_{z}^{c \sigma}- \\
-m g+2 N_{B 1}(\sin \alpha \cos \varepsilon+f \sin \varepsilon)+ \\
+N_{K}(\sin \gamma-f \cos \gamma) .
\end{gathered}
$$

The system of differential equations $(32-34)$ allows determining the values of dynamic reactions at contact points $\bar{N}_{K}, \bar{N}_{B 1}$ and of the rotational moment $M^{\gamma}$ dependent on the angle $\gamma$.

Determination of dynamic reactions at contact points was done numerically with the help of the software Mathcad for both cases (i) and (ii). The algorithm for the problem solution was developed based on equations $(33,34)$ in case (i), and equations (29-31) in case (ii). The algorithm allows the assembly mode and dimensions of the aligned parts to be chosen. In this work the simulation results for the alignment with the following set of the components' dimensions are presented: $D=50 \mathrm{~mm}, d=49.95 \mathrm{~mm}, H=70 \mathrm{~mm}$. The angular velocities of the constituent motions here were taken to be constant: $\dot{\gamma}=21 / \mathrm{s}, \dot{\psi}=10$ $1 / \mathrm{s}$, and friction factor is $f=0.2$. The solution for the components with the indicated dimensions is presented at Fig. 9 in the form of plots of the dependence of $N_{1}=\frac{N_{B 1}}{m g}$ on angle $\gamma$. This value $N_{1}$ represents the ratio of normal reaction to the peg gravity at one of the symmetric contact points $B_{1}$. Here the dashed line represents case (i) with $\dot{\psi}_{1}=0$, and the solid line represents case (ii) with $\dot{\psi}_{2}=10 \mathrm{~s}^{-1}$.

From the graph we can see that in both cases the forces acting on the peg contact points increase during alignment, with the significant change near the value $\gamma_{O}=\arccos \frac{d}{D}$. At this angle the symmetric contact points merge at the symmetry axis into one point, thus turning a three-point contact into a two-point contact. Near this point the value $N_{1}$ for case (i) significantly exceeds the peg gravity force, which can cause the jamming of the components or the damaging of their surfaces. By comparing the forces $N_{1}$ for the cases (i) and (ii) it is quite distinctive that the rotational movement in case (ii) significantly reduces the interaction forces, which improves the conditions of the parts aligning and reduces the probability of jamming.

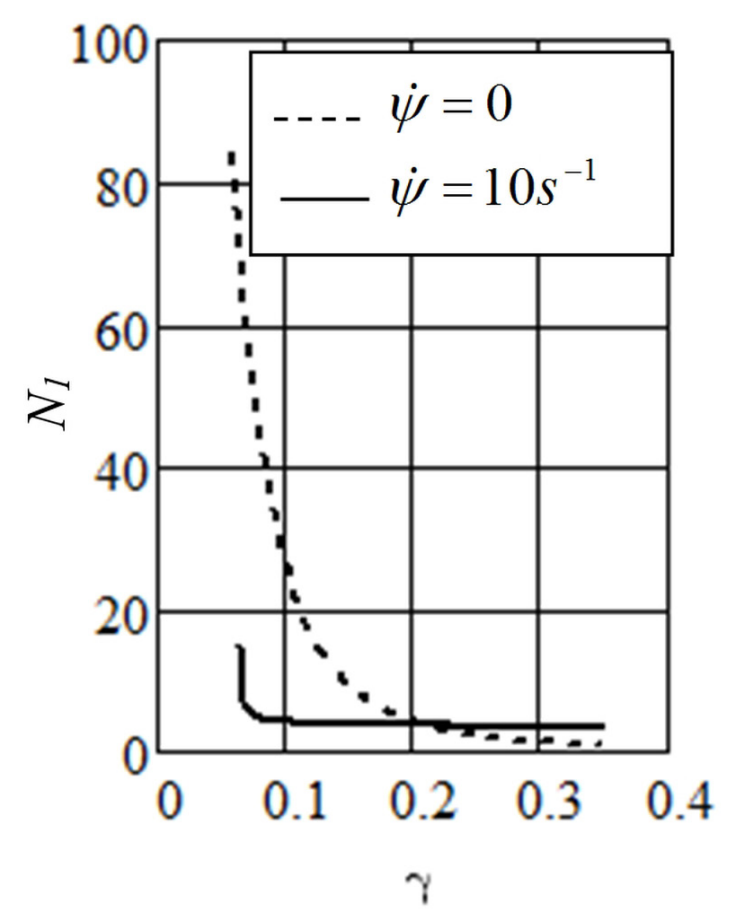

Fig. 9. Dependence of normal re-action at point B1 upon angular velocity of rotation around the ax-is of the hole. 


\section{CONCLUSIONS}

The detailed kinematic analysis of the peg's complex movement while leaning on the edge of the vertically fixed hole, with two degrees of freedom in the course of cylindrical parts alignment was performed. The directions of the interaction forces at points of contact between the peg and the hole were determined. The Dynamical Differential Equations describing the process of alignment of the components in dependence on their relative location, acting forces and taking the effect of a rotational movement on this process in consideration were set up.

The usability and the power of the Dynamical Differential Equations was demonstrated by the study of two simple cases of alignment of the components. This study showed that an additional rotation of the peg around its axis could decrease the risk of jamming and the surface damage during the alignment.

In general, the mathematical model of the peg and hole alignment based on Dynamical Differential Equations, represented in this paper, allows a more tailored and thorough application and can be a powerful tool for the very broad spectrum of technical tasks extending the technological possibilities of robotic assembly of the cylindrical parts, including selection of parameters and modes of assembly, development of assembly devices as well as computer modeling extending the technological possibilities of robotic assembly of the cylindrical parts.

In this paper the peg-on-hole assembly was considered in two Euler angles: nutation angle $\gamma=\gamma(t)$, and the precession angle $\psi=\psi(t)$. In the future work another combination of Euler angles is planned to be considered: the alignment angle $\gamma=\gamma(t)$ and the rotation of the peg around its own axes $\varphi=\varphi(t)$.

\section{Acknowledgements}

The authors express deep gratitude to Philip Peterson from the Department of Physics and Astronomy of University of Leicester for a thorough proofreading of the paper. He improved the level of English language and suggested valuable corrections in use of technical terms.

\section{REFERENCES}

1. Abu-Dakka F., Nemec B., and Ude A., Peg-In-Hole using Dynamic Movement Primitives, Proceedings of RAAD, the 21th International Workshop on Ro- botics in Alpe-Adria-Danube Region, Napoli, Italy 2012, 143-149.

2. Bakšys B. B., Chadarovičius A., and Pilkauskas K., Experimental research of parts vibratory alignment with remote center compliance device, Journal of Vibroengineering, 11(2), 2009, 226-232.

3. Bozhkova L. V., Vartanov M. V., Zimina I. N., Mathematical model of robotic assembly with application of adaptation and low-frequency oscillations, Sborka v mashinostroenii i priborostrenii [Assembly in machine-building and instrument making], 16, 2015, 16-20.

4. Bruyninckx H., Dutrd S. and De Schuttes J., Pegon-Hole: A Model Based Solution to Peg and Hole Alignment, Proceedings of the IEEE International Conference on Robotics and Automation, Nagoya, Japan 1995, 1919-1924

5. Chernyakhovskaya L. B., Simakov D. A. Kinematics and dynamics of the process of cylindrical parts assembly, Sborka v mashinostroenii i priborostrenii [Assembly in machine-building and instrument making], 1, 2012, 27-32.

6. Ivanov A. A, Analytical conditions of automatic assembly, Sborka v mashinostroenii i priborostrenii [Assembly in machine-building and instrument making], 8, 2009, 13-17.

7. Kholodkova A. G, Peculiarities of automatic performance of cylindrical connections with small gaps, Sborka $\mathrm{v}$ mashinostroenii i priborostrenii [Assembly in machine-building and instrument making], 4, 2004, 14-18.

8. Kremleva N. G., Bezrukova T. B. Research of the process of automatic rotational assembly of cylindrical parts, Sborka v mashinostroenii i priborostrenii [Assembly in machine-building and instrument making], 6, 2015, 29-32.

9. Kristal M. G., Chuvilin I. A., Model of rotational assembly dynamics of cylindrical parts, Sborka v mashinostroenii i priborostrenii [Assembly in machinebuilding and instrument making], 8, 2008, 12-15.

10. Puente E. A., Balaguer C. and Barrientos A., Forcetorque sensor-based strategy for precise assembly using ASCARA robot, Robotics and Autonomous System, 8, 1991, 203-212.

11. Savarimuthu T. R. et al., Analysis of human Pegin-Hole Executions in a Robotic Embodiment using uncertain Grasps, Proceedings of the 9th International Workshop on Robot Motion and Control, Wasowo, Poland 2013, 233-239.

12. Zamyatin V. G, Geometrical conditions of assemblability at automatic assembly of cylindrical connections with the gap, Izvestiya vuzov, Mashinostroenie [News of high schools, Machine-building], 12, 1972, 162-166.

13. Zharkov G. E. Assembly method by changing the position of gravity center, Sborka $\mathrm{v}$ mashinostroenii i priborostrenii [Assembly in machine-building and instrument making], 11, 2009, 7-9. 